\title{
Characterization of neutron emission during pulse mode of low output electronic neutron generator
}

\author{
Tomas Bily ${ }^{1}$, Ondrej Huml ${ }^{1}$ \\ ${ }^{1}$ Czech Technical University in Prague, Faculty of Nuclear Sciences and Physical Engineering, \\ Department of Nuclear Reactors, V Holesovickach 2, Prague 8 \\ tomas.bily@fjfi.cvut.cz
}

\begin{abstract}
The paper deals with the characterization of the neutron output during operation the neutron generator in the pulsing mode. The characterization is performed by a cadmium covered He-3 detector. First, the adequacy of a cadmium covered He-3 detector to study the pulsed mode operation of neutron generator is assessed using Monte Carlo simulations in MCNP6 code. Second, the pulsing mode operation of the D-D P-385 Thermo Fisher Scientific neutron generator with a maximal neutron output of $7 \times 106 \mathrm{n} / \mathrm{s}$ is studied. The study shows the dependence of neutron emission and pulse shapes on the frequency, duty factor, high voltage and beam current.
\end{abstract}

Keywords - portable neutron generators; pulse characterisation.

\section{INTRODUCTION}

$\mathrm{P}$ ORTABLE electronic neutron generators are an established technology that can be used for many lab and field applications. Offering significant regulatory and operational benefits over radionuclide neutron sources, they start to be more widely used at universities and research centres. Beside the established industrial applications (e.g., in logging or mining industry), applications are being developed for fields including security, safeguards, or waste characterization. They are becoming more used for E\&T in nuclear engineering, nuclear analytical techniques, or nuclear related fields. They can be utilized for many analytical techniques (including NAA, PGNAA, DNC, DDA). In many cases, the pulsed operation of the generator is required or can be used to improve the efficiency of the applied method. Consequently, the knowledge on the actual production of neutron and its dependence on operational parameters is important.

The paper provides such characterization performed by a cadmium covered He-3 detector. First, the adequacy of cadmium covered He-3 detector to study the pulsed mode operation of neutron generator is assessed utilizing Monte Carlo simulations in MCNP6 code. Second, the pulsing mode operation of the D-D P-385 Thermo Fisher Scientific neutron generator is analyzed.

\section{MATERIALS AND METHODS}

\section{A. P-385 neutron generator}

The $\mathrm{P}-385$ is a D-D portable neutron generator produced by Thermo Scientific [1]. It has maximum emission rate of $7 \times 10^{6}$ $\mathrm{n} / \mathrm{s}$ [2]. It can be operated in continuous or pulsed mode with frequency up to $20 \mathrm{kHz}$ with adjustable duty cycle between $5 \%$ and $100 \%$ ( $5 \mu$ s pulse width minimum). The neutron output of the generator can be adjusted by set-up of high voltage (up to $130 \mathrm{kV}$ ) and beam current (up to $70 \mu \mathrm{A}$ ).

The generator can provide information on neutron production via a TTL signal that can be used for triggering the data acquisition system. Within this paper the SOURCE (synchronized) signal was used. The SOURCE pulse drives the ion source pulse of the generator. As stated by the producer, there is a slight delay due to the source capacitance charging before a neutron pulse is generated. This delay varies based on the beam current and pulse width settings [1]. Besides the SOURCE signal, three other DELAY signals are available [1].

\section{B. Detector and data acquisition}

Measurements were performed with He-3 chamber DEXTRAY 65NH45 (length/diameter 650/25 mm) and TEMA EMK-310 analyzer. A system based on a programmable FPGA was developed to collect data from the neutron detector, which are time-synchronized with the pulse mode of D-D neutron generator operation (i.e. synchronized with the SOURCE signal). The system is based on the RedPitaya STEMLAB 12514 platform containing the Xilinx Zynq 7010 FPGA. This platform contains up to 16 digital input/output ports. These are used for data collection purposes as inputs for fast independent counters implemented inside the FPGA. The first counter monitors the signal on start of neutron production in each cycle of the generator. It assures the synchronization of the measurement cycles of data collection with the pulse cycles of the generator. The developed software allows to divide the neutron response in each cycle into 1000 time-bins. At a D-D generator frequency of the order of hundreds $\mathrm{Hz}$, it is then possible to achieve a time resolution of the order of tens of $\mu \mathrm{s}$. Good statistical response can then be achieved by repeating a large number of cycles and accumulating results.

For the measurements the detector was placed inside a coverage made by cadmium to cut the contribution of thermal neutrons 
and thus to approach the time dependency of the detector response to the time dependency of neutron emission.

\section{Experimental set-up}

The experimental set-up is shown in the Fig. 1 and 2. It is used for experiments with radionuclide neutron sources and electronic neutron generators [3]. The neutron generator was placed inside a central channel of the polyethylene box of $50 \mathrm{x}$ $50 \mathrm{~cm} \times 100 \mathrm{~cm}$, the cadmium covered detector was placed in one of the dry experimental channels with diameter of $7 \mathrm{~cm}$, the centre of the detectors was located $10.5 \mathrm{~cm}$ from the centre of the tube. The measurements were performed for duty factors ranging from $10 \%$ to $50 \%$ and with pulsing frequencies from $100 \mathrm{~Hz}$ to $500 \mathrm{~Hz}$, i.e. for rather lower part of the frequency range, starting even below the minimum stated frequency of $250 \mathrm{~Hz}$. Such low frequencies can be used e.g. for delayed neutron counting applications (see e.g. [4]) Measurements with various high voltage and beam current have been performed to show the behaviour at maximum tube parameters $(130 \mathrm{kV}$ and $70 \mu \mathrm{A})$ and at lower ones.

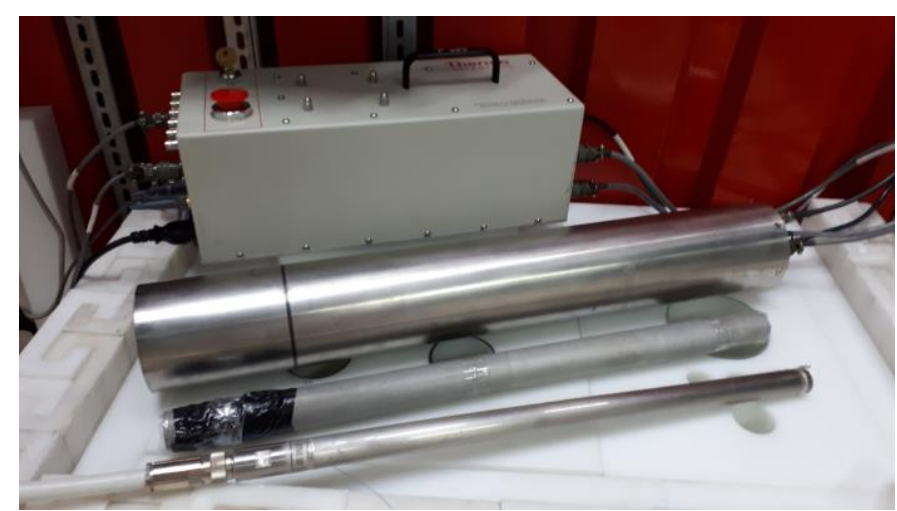

Fig. 1. Neutron generator control unit, generator tube, cadmium coverage and He-3 detector used in the study on top of polyethylene block

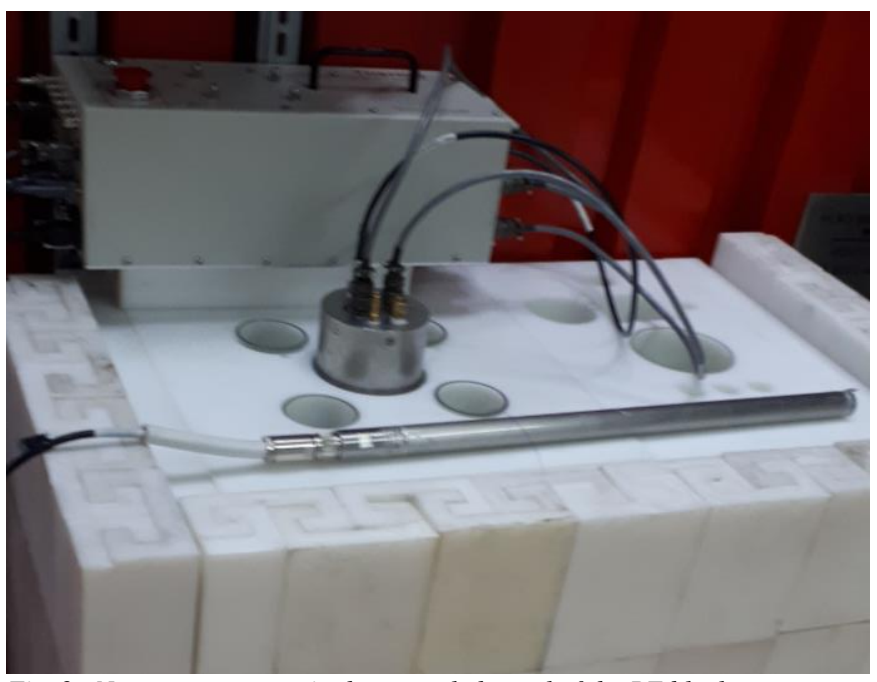

Fig. 2. Neutron generator in the central channel of the PE block

\section{Calculations}

Calculations in Monte Carlo code MCNP6 [5] have been performed to prove the adequacy and to assess the limitations of cadmium covered thermal neutron detector to study timedependent behavior of neutron generator pulsed mode operation. The neutron source was simulated using SDEF card of the MCNP as a point isotropic source with the energy of 2.45 $\mathrm{MeV}$. All neutrons have been set to start at time equal to zero. The calculated quantities included the time-dependent neutron flux and detector response. For comparison both the case of cadmium covered and bare He-3 detectors were included. The tallies were binned to understand the contribution of neutrons below $1 \mathrm{eV}, 1 \mathrm{eV}-1 \mathrm{MeV}$, and above $1 \mathrm{MeV}$. The $1 \mathrm{eV}$ boundary was chosen due to its closeness to the cadmium edge. The quantity corresponding to detector response was calculated as $\mathrm{He}-3(\mathrm{n}, \mathrm{p}) \mathrm{H}-3$ reaction rate. Two time-binning schemes were used to observe fast and slower part of the response. The former calculated the response in the first $50 \mu$ s with the time step of $0.1 \mu \mathrm{s}$, the latter in first $5 \mathrm{~ms}$ with the time step of $10 \mu \mathrm{s}$. Such calculations can describe the time scales between the emission of neutron by generator and reaction occurring in the detector. The further delays caused by charge collection time in detector or those caused by electronic are neglected here. All calculations were performed with the ENDF/B-VIII nuclear data library [6]. Ca $2 \times 10^{8}$ initial neutrons were used for each simulation.

The geometry is shown in the Fig. 3. The time dependent response of neutron detectors has been calculated both for bare $\mathrm{He}-3$ detector, and cadmium covered $\mathrm{He}-3$ detector.

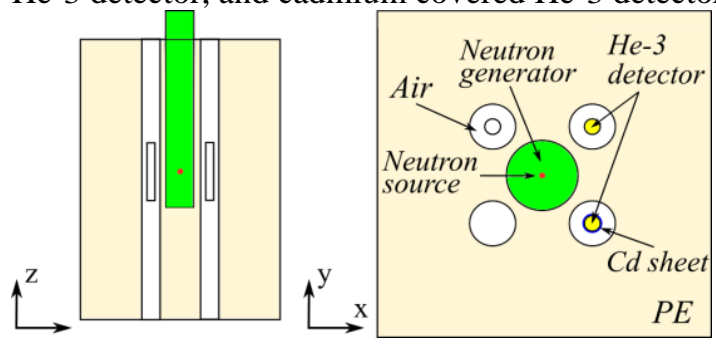

Fig. 3. Geometry used in calculations

The neutron generator was placed to the centre of the geometry. The generator internals were modelled in detail based on the input provided by the tube producer. The detectors assumed in calculations were He-3 tubes with active length of $20 \mathrm{~cm}$, diameter of $2.5 \mathrm{~cm}$, and with helium pressure in the tube of 2 bars. The centre of the detectors was placed $10.5 \mathrm{~cm}$ from the centre of the tube (neutron source position); vertically the centre of the detector corresponds to the source plane. Only active volumes of the detectors were simulated. In case A (moderated geometry), the generator was placed inside PE block of $50 \times 50$ $\mathrm{x} 100 \mathrm{~cm}$; the detectors were placed in dry channels with diameter of $7 \mathrm{~cm}$. One detector was simulated as bare, another was covered by $1 \mathrm{~mm}$ thick cadmium sheet with density of 8.65 $\mathrm{g} / \mathrm{cm}^{3}$.

\section{RESULTS OF CALCULATIONS}

The results are shown in Figs. 4-6. Fig. 4 shows the timeevolution of neutron flux. In the first $5 \mu \mathrm{s}$, the flux is dominated by neutrons above $1 \mathrm{eV}$, after that, the neutrons below $1 \mathrm{eV}$ became dominant. Initially, the response of bare He-3 detector (see Fig. 5) drops sharply reaching minimum around $5 \mu \mathrm{s}$, then it starts to rise due to onset of neutrons below $1 \mathrm{eV}$, and their high reaction cross-section. The maximum response is achieved around $30 \mu \mathrm{s}$ after neutron emission from the generator. Then, the response starts to decrease slowly. For detector covered in 
cadmium (see Fig. 6) the low energy neutrons are efficiently cut and the response drops in less than $15 \mu \mathrm{s}$ to $1 \%$ of its value at time 0 (for response evaluated with time step of $0.1 \mu \mathrm{s}$ ).

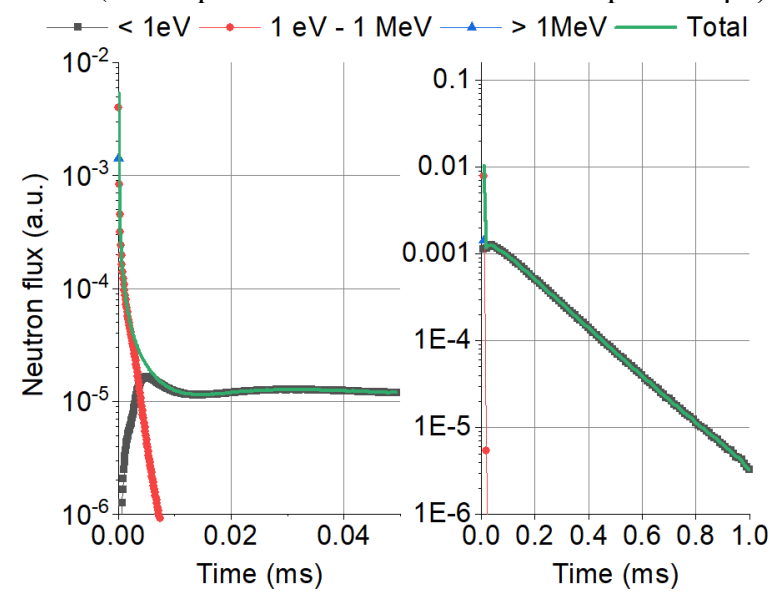

Fig. 4. Neutron flux evolution at detector location of moderated geometry. Left: short term behavior (time step of $0.1 \mu \mathrm{s}$ ); right: long term behavior (time step of $10 \mu \mathrm{s})$

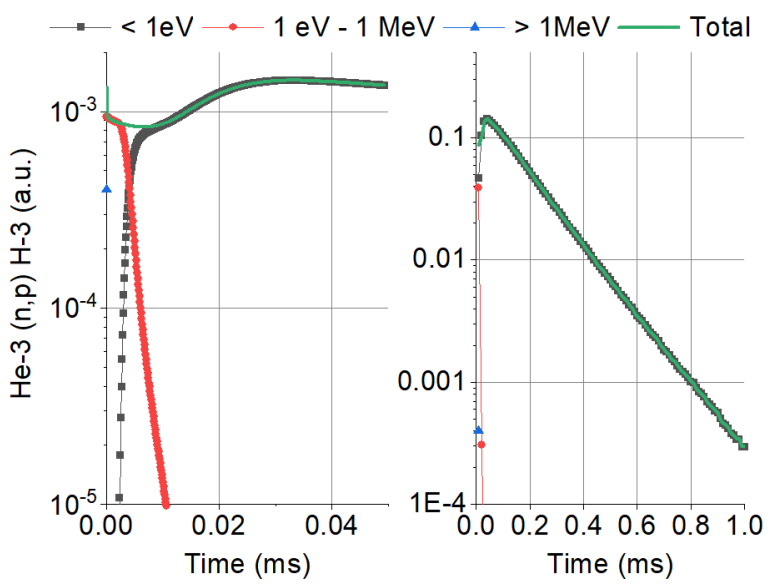

Fig. 5. Relative detector response of bare detector in moderated geometry. Left: short term behavior (time step of $0.1 \mu \mathrm{s}$ ); right: long term behavior (time step of $10 \mu \mathrm{s})$

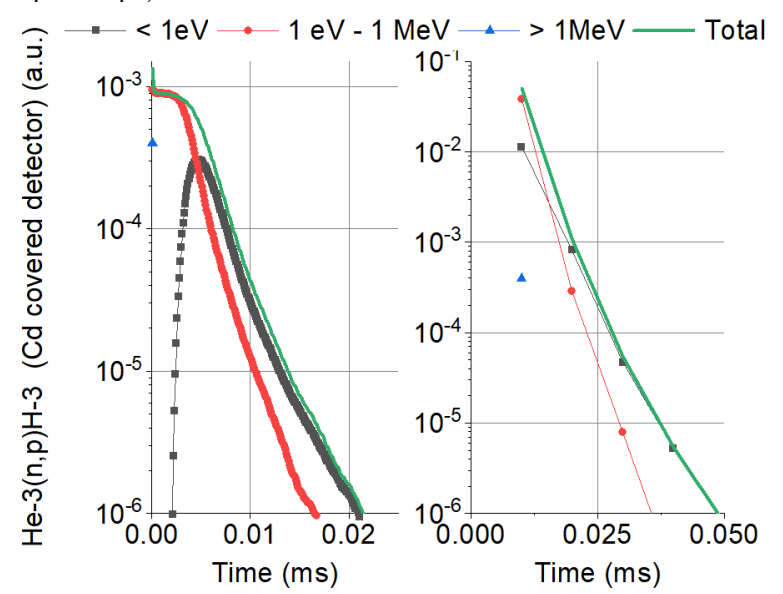

Fig. 6. Relative detector response of $\mathrm{Cd}$ covered detector in moderated geometry. Left: short term behavior (time step of $0.1 \mu \mathrm{s}$ ); right: long term behavior (time step of $10 \mu \mathrm{s}$ )

Based on the calculations, one can conclude that characterisation of neutron generator pulse mode behaviour by $\mathrm{Cd}$-covered $\mathrm{He}-3$ detector in moderated geometry is reasonable, and especially for lower range of frequencies (say below $1 \mathrm{kHz}$ ) it can provide precise information on pulse shape. For higher frequencies the applicability of the approach would be limited as the response delay after the neutron emission from the generator might form a significant part of the cycle.

\section{RESULTS OF MEASUREMENTS}

The following section summarizes the measurements performed with the Cd-covered He-3 detector and P-385 neutron generator to characterize its pulse mode operation. The characteristics includes the generator start-up, pulse stability, total neutron output dependence on pulsing set-up, and neutron emission pulse shape dependence on pulsing set-up.

\section{A. Generator start-up \& pulse stability}

The start-up of neutron generator for various set-ups is illustrated in the Fig. 7. In the figure, the time evolution starts when the first pulses have been detected. One may observe the generator reaches its nominal output ca. 5 to 8 seconds after the start-up, first 3 to 5 seconds producing only negligible number of neutrons. The rise time seems to be more dependent on duty factor set-up than on high voltage and beam current set-up.

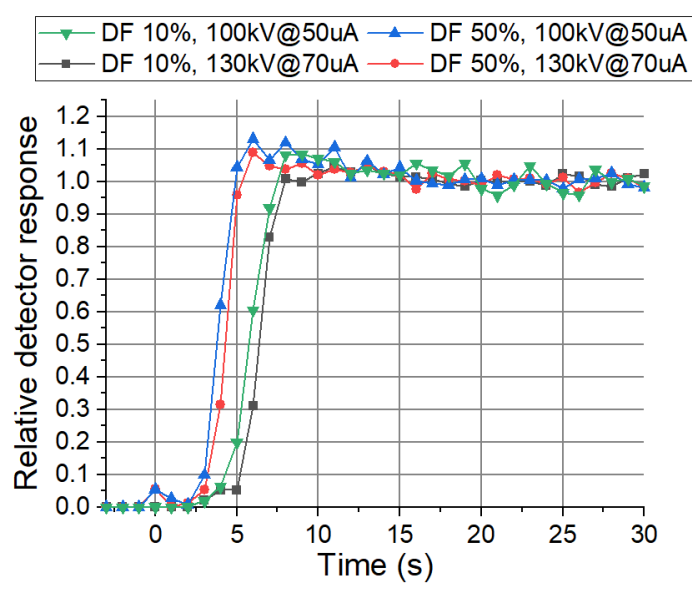

Fig. 7. Relative detector response of Cd-covered detector in moderated geometry to generator start-up for four set-ups at $250 \mathrm{~Hz}$.

The pulse stability has been observed for five-minute operation of the tube with various set-ups of pulsing frequencies, duty factors, high voltages, and beam currents. The detector response was summed per five seconds, thus creating 60 batches per each five-minute-long run. The results are given in the figure 8 . One can observe typically one or two batches with lower neutron output that correspond to the generator start-up. Then, the pulse shapes remained stable throughout the operation.

\section{B. Neutron output dependence on pulsing set-up}

The generator pulsing set-up may affect the neutron emission rate. The dependence on pulsing frequency and beam current, measured as number of neutron counts detected over five minute operation, is illustrated in the figure 9 for three frequencies and several values of duty factor of generator operating at nominal high voltage and beam current $(130 \mathrm{kV}$, $70 \mu \mathrm{A})$. Results were normalized to the value obtained at 250 $\mathrm{Hz}$ with duty factor of $10 \%$. Figure 10 shows the neutron output dependence on high voltage and beam current at two fixed values of duty factor at frequency of $250 \mathrm{~Hz}$. Obviously, the 
neutron emission rises linearly with beam current with the slope given by high voltage and duty factor set-up.
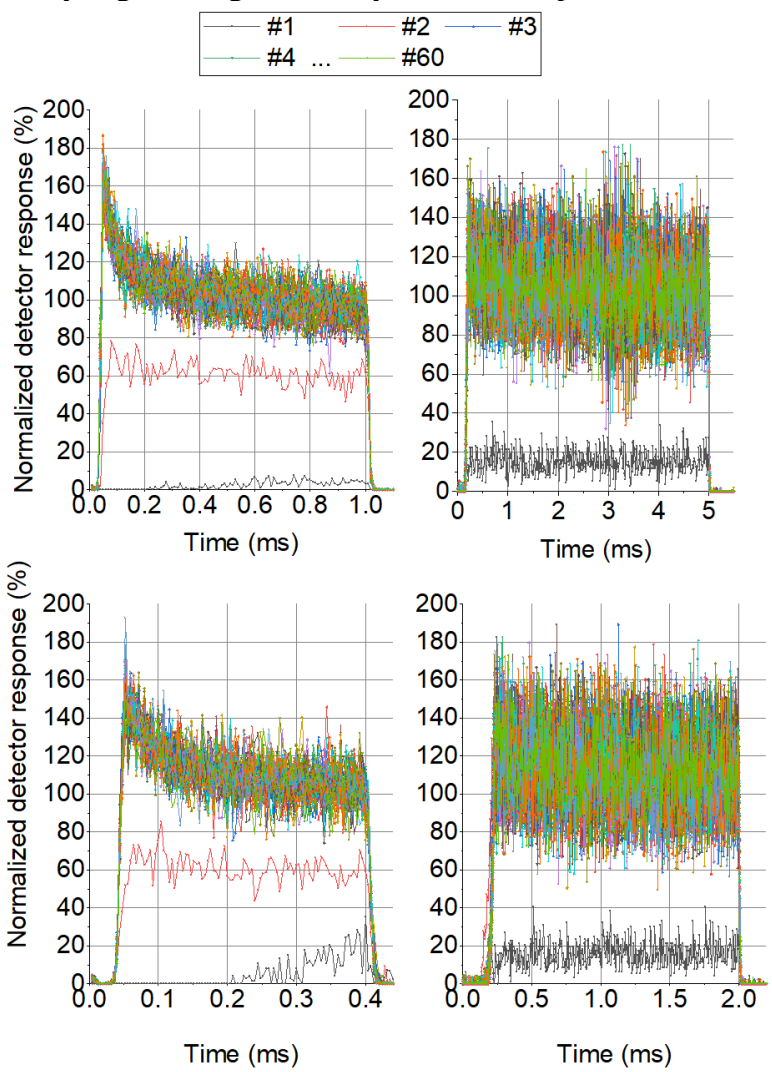

Fig. 8 Cadmium-covered detector response to P-385 D-D neutron generator operated in pulsed mode. Counts integrated per $5 \mathrm{~s}$ for 5 minutes of operation at $130 \mathrm{kV} @ 70 \mu \mathrm{A}$ (i.e. 60 batches denoted as \#1 to \#60. For $100 \mathrm{~Hz}, 1$ batch sums over 500 cycles, for $250 \mathrm{~Hz}$ one batch sums over 1250 cycles). Top left: $100 \mathrm{~Hz}, \mathrm{DF} 10 \%$. Top right: $100 \mathrm{~Hz}, \mathrm{DF} 50 \%$. Bottom left: $250 \mathrm{~Hz}, \mathrm{DF} 10 \%$. Bottom right: $250 \mathrm{~Hz}, \mathrm{DF} 50 \%$.

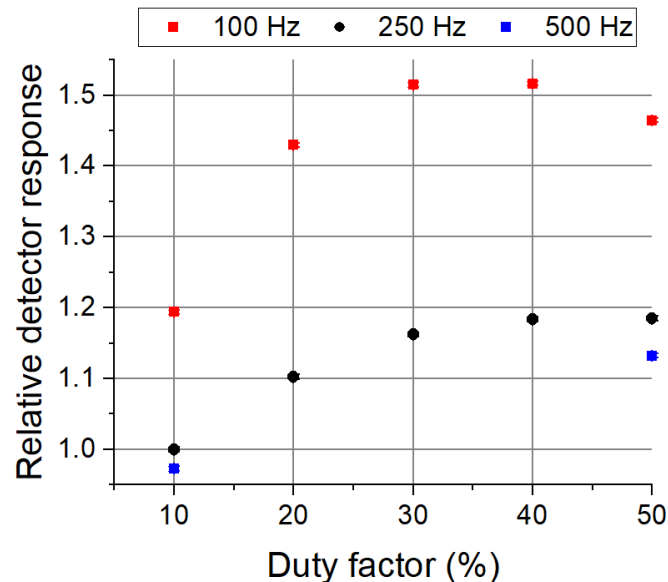

Fig. 9 Cadmium-covered detector response to P-385 D-D neutron generator operated in pulsed mode demonstrating the neutron output dependence on pulsing frequency and duty factor at constant high-voltage and beam current
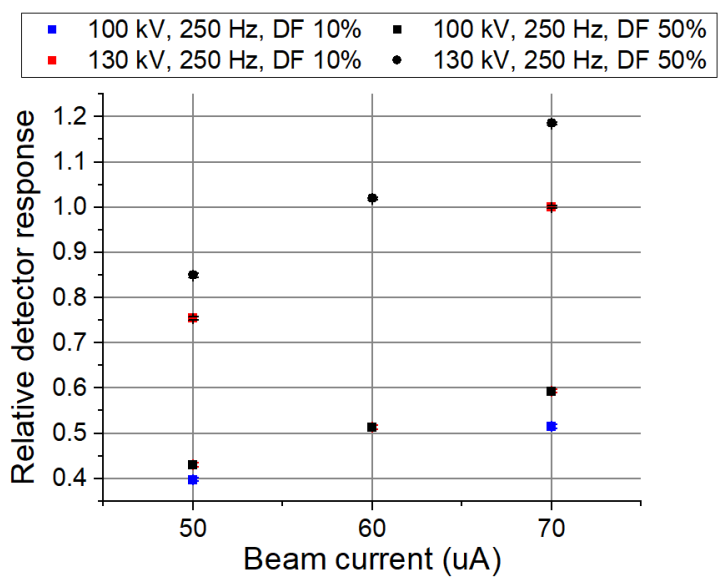

Fig. 10 Cadmium-covered detector response to P-385 D-D neutron generator operated in pulsed mode demonstrating the neutron output dependence on beam current

\section{Neutron emission pulse shape dependence on pulsing set- up}

The neutron emission pulse shapes for two frequencies were observed at nominal high voltage and beam current parameters $(130 \mathrm{kV}, 70 \mu \mathrm{A})$ for two frequencies and five values of duty factor (see Fig. 11). For all studied cases deviations from idealized rectangular shape can be observed. At these highvoltage and beam current set-ups, the neutron emission was higher at the pulse start and decreased to a steady value. This effect was stronger for lower values of duty factors. On the other hand, the higher was the duty factor, the longer took the pulse rise-time, reaching more than $0.2 \mathrm{~ms}$ for duty factor of 50 $\%$ and frequency of $250 \mathrm{~Hz}$.

Fig. 12 shows the comparison of pulse shapes at $250 \mathrm{~Hz}$ for two fixed values of duty factors for varying high voltage and beam current. One can see that the emission rise time differ slightly for various set-ups, being ruled mostly by the beam current. Contrary, at the end of the pulse, the emission drops rapidly and practically identically for all studied set-ups. Further, one can observe that for high values of duty factor (here DF $=50 \%$ ), the pulse is nearly rectangular whereas for low values of duty factor (here DF $=10 \%$ ), the pulse shape depends strongly on applied high voltage. Finally, Fig. 13 provides the comparison of neutron emission pulse shapes for three frequencies and two values of duty factor. The time scale corresponds to percent of a cycle. One can see the neutron emission delays after the indicated pulse start (SOURCE signal driving the ion source). This is more relevant for higher values of duty factor and higher frequencies, e.g., for the frequency of $500 \mathrm{~Hz}$ at duty factor of $50 \%$, the pulse started after $10 \%$ of the cycle time (i.e. after $0.2 \mathrm{~ms}$ ). At $250 \mathrm{~Hz}$, the delay was similar (ca. $0.2 \mathrm{~ms}$ ) but related to cycle length it accounts to only $5 \%$ of the cycle length. 

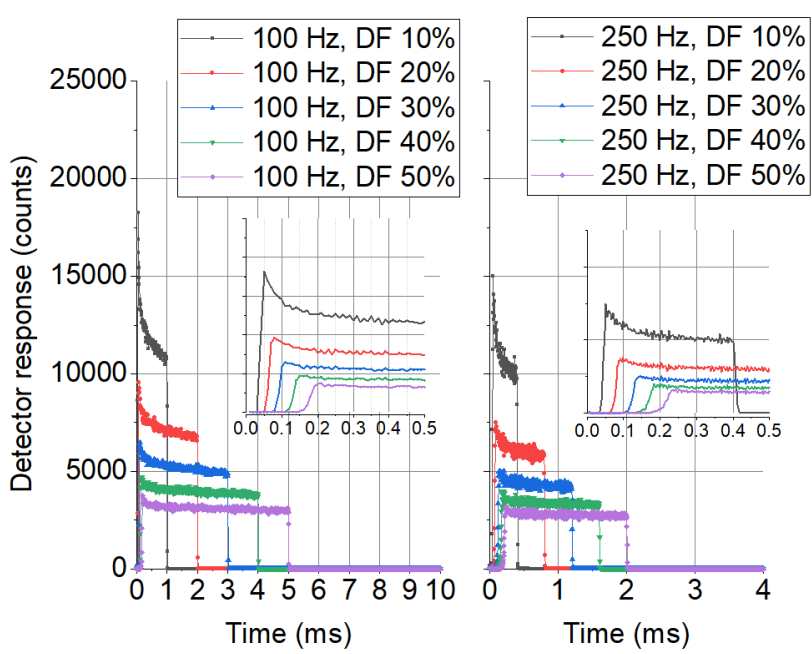

Fig. 11 Cadmium-covered detector response to P-385 D-D neutron generator operated at $130 \mathrm{kV}$ and $70 \mu \mathrm{A}$ for pulsed operation at $100 \mathrm{~Hz}$ and $250 \mathrm{~Hz}$ with duty factor ranging from $10 \%$ to $50 \%$.

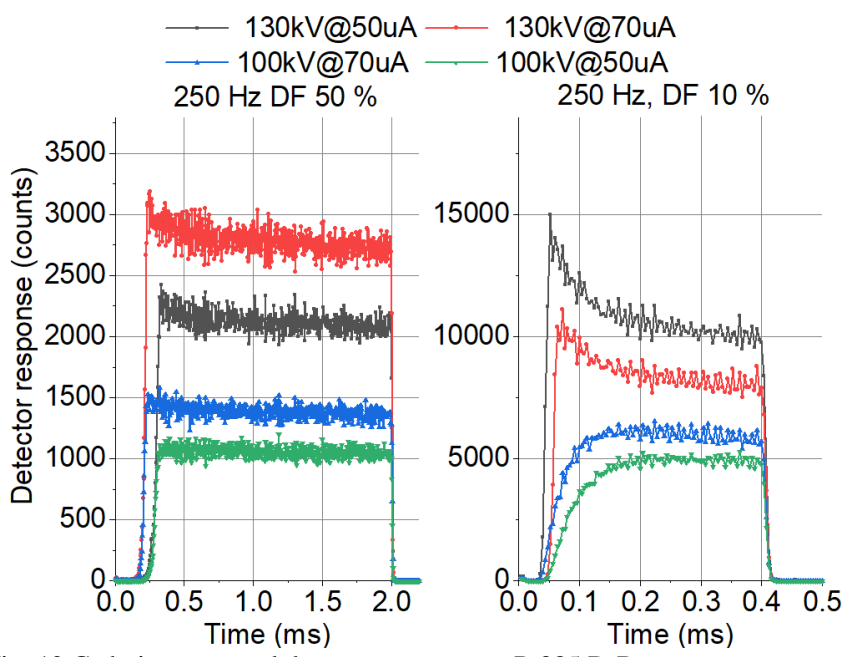

Fig. 12 Cadmium-covered detector response to P-385 D-D neutron generator operated in pulsed mode at $250 \mathrm{~Hz}$ with duty factor ranging from $10 \%$ to 50 $\%$ with varying high voltage and beam current setup.

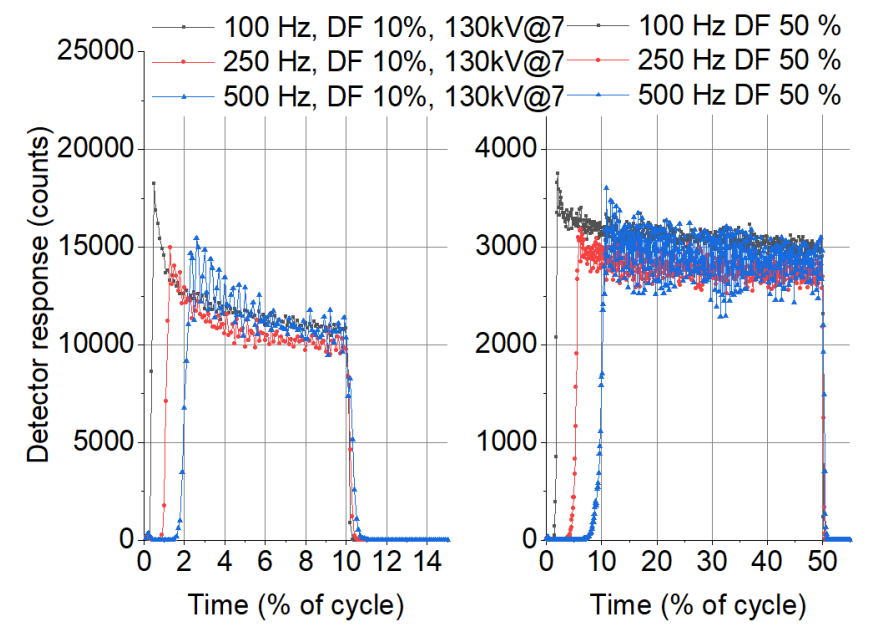

Fig. 13 Cadmium-covered detector response to P-385 D-D neutron generator operated in pulsed mode. Comparison of neutron emission pulse shapes for three frequencies and two values of duty factor at $130 \mathrm{kV} @ 70 \mu \mathrm{A}$.

\section{CONCLUSIONS}

The paper deals with the characterization of low-output neutron generator pulsed mode operation. First, the calculations performed in the MCNP6 code proved the viability to use He-3 thermal neutron detector covered by cadmium for the purpose, especially for lower frequencies (say below $1 \mathrm{kHz}$ ). Further, various aspects of neutron production in pulsed mode were demonstrated for P-385 neutron generator operated between $100 \mathrm{~Hz}$ and $500 \mathrm{~Hz}$, duty factors between $10 \%$ and $50 \%$ and for various set-ups of high voltage and beam current. This might help to improve the utilization of the generator in applications utilizing the generator pulsed mode.

\section{ACKNOWLEDGMENT}

This work was supported by the project "Utilisation of radiation-based methods for detection and identification of CBRNE materials" (project nr. VI20172022171) supported by Ministry of the Interior of the Czech Republic.

\section{REFERENCES}

[1] Thermo Fisher Scientific Inc., "P 385 Neutron Generator Operation Manual". Tech. Rep. Manual P/N 120006-A 062907, 2010.

[2] J. Rataj, T. Bílý, O. Huml, et al., "Verification of the MCNP model of Manganese Bath," Prague, 2020 21 st International Scientific Conference on Electric Power Engineering (EPE), Czech Republic 2020, doi: 10.1109/EPE51172.2020.9269226

[3] J. Rataj, P. Suk, T. Bílý, et al., "Characterisation of neutron field in the polyethylene neutron irradiator", Applied Radiation and Isotopes, vol. 168, 2021. DOI. 10.1016/j.apradiso.2020.109529

[4] T.-H. Lee, H.-S. Shin, and H.-D. Kim, "Delayed Neutron Measurement in High Frequency Mode and Its Application to the Determination of Fissile Content in Spent Fuel Assembly", Valencia, 2011 IEEE Nuclear Science Symposium Conference Record N53-7, Spain, 2011

[5] C.J. Werner, et al., "MCNP6.2 Release Notes". Los Alamos National Laboratory, report LA-UR-18-20808, 2018.

[6] D.A.Brown, M.B.Chadwick, R.Capote, et al., "ENDF/B-VIII.0: The 8th major release of the nuclear reaction data library with CIELO-project cross sections, new standards and thermal scattering data", Nucl. Data Sheets 148(2018)1, DOI. 10.1016/j.nds.2018.02.001 